\title{
Exploring the singlet scalar dark matter from direct detections and neutrino signals via its annihilation in the Sun
}

\author{
Wan-Lei Gud* and Yue-Liang $\mathrm{Wu}$ \\ Kavli Institute for Theoretical Physics China, \\ Key Laboratory of Frontiers in Theoretical Physics, \\ Institute of Theoretical Physics, Chinese Academy of Science, Beijing 100190, China
}

\begin{abstract}
We explore the singlet scalar dark matter (DM) from direct detections and high energy neutrino signals generated by the solar DM annihilation. Two singlet scalar DM models are discussed, one is the real singlet scalar DM model as the simple extension of the standard model (SSDM-SM) with a discrete $Z_{2}$ symmetry, and another is the complex singlet scalar DM model as the simple extension of the left-right symmetric two Higgs bidoublet model (SSDM-2HBDM) with $P$ and $C P$ symmetries. To derive the Sun capture rate, we consider the uncertainties in the hadronic matrix elements and calculate the spin-independent DMnucleon elastic scattering cross section. We find that the predicted neutrino induced upgoing muon fluxes in the region $3.7 \mathrm{GeV} \leq m_{D} \leq 4.2 \mathrm{GeV}$ slightly exceed the Super-Kamiokande limit in the SSDM-SM. However, this exceeded region can be excluded by the current DM direct detection experiments. For the SSDM-2HBDM, one may adjust the Yukawa couplings to avoid the direct detection limits and enhance the predicted muon fluxes. For the allowed parameter space of the SSDM-SM and SSDM-2HBDM, the produced muon fluxes in the Super-Kamiokande and muon event rates in the IceCube are less than the experiment upper bound and atmosphere background, respectively.

PACS numbers: 95.35.+d, 12.60.-i, 95.55.Vj, 13.15.+g
\end{abstract}

*Email: guowl@itp.ac.cn

†Email: ylwu@itp.ac.cn 


\section{INTRODUCTION}

The existence of dark matter (DM) is by now well confirmed [1, 2]. The recent cosmological observations have helped to establish the concordance cosmological model where the present Universe consists of about $73 \%$ dark energy, $23 \%$ dark matter and $4 \%$ atoms [3]. Understanding the nature of dark matter is one of the most challenging problems in particle physics and cosmology. Currently, many DM search experiments are under way. These experiments can be classified as the direct DM searches and the indirect DM searches. The direct DM detection experiments may observe the elastic scattering of DM particles with nuclei. The indirect DM searches are designed to detect the DM annihilation productions, which include neutrinos, gamma rays, electrons, positrons, protons and antiprotons. In addition, the collider DM searches at CERN LHC are complementary to the direct and indirect DM detection experiments.

The indirect DM searches are usually independent of the direct DM searches. Namely, one can calculate the DM annihilation signals when the thermal-average of the annihilation cross section times the relative velocity $\langle\sigma v\rangle$ and the DM annihilation productions are known. It is worthwhile to stress that the DM annihilation signals from the Sun (or Earth) depend on both the direct DM detection and the indirect DM detection. When the DM particles elastically scatter with nuclei in the Sun, they may lose most of their energy and are trapped by the Sun [1]. The solar DM capture rate is related to the DM-nucleon elastic scattering cross section. These trapped DM particles will be accumulated in the core of the Sun due to repeated scatters and the gravity potential. Therefore the Sun is a very interesting place for us to search the DM annihilation signals [4-9]. The DM annihilation rate in the Sun depends on $\langle\sigma v\rangle$ and the solar DM distribution. If the DM annihilation rate reaches equilibrium with the DM capture rate, the solar DM annihilation rate only depend on the DM-nucleon elastic scattering cross section. Due to the interactions of the DM annihilation products in the Sun, only the neutrino can escape from the Sun and reach the Earth. These high energy neutrinos interact with the Earth rock or ice to produce upgoing muons which may be detected by the water Cherenkov detector Super-Kamiokande (SK) [10] and the neutrino telescope IceCube [11].

In this paper, we explore the singlet scalar dark matter from direct detections and high energy neutrino signals via the solar DM annihilation in two singlet scalar DM models. One is the real singlet scalar DM model as the simple extension of the standard model (SSDM-SM) [12 16] and another is the complex singlet scalar DM model as a simple extension of the left-right symmetric 
two Higgs bidoublet model (SSDM-2HBDM) [17-19]. In the SSDM-SM, a real singlet scalar $S$ with a $Z_{2}$ symmetry is introduced to extend the standard model. Although this model is very simple, it is phenomenologically interesting [12-16]. In the SSDM-2HBDM, the imaginary part $S_{D}$ of a complex singlet scalar field $S=\left(S_{\sigma}+i S_{D}\right) / \sqrt{2}$ with $P$ and $C P$ symmetries can be the DM candidate [19]. The stability of $S_{D}$ is ensured by the fundamental symmetries $P$ and $C P$ of quantum field theory. In Refs. [16] and [19], we have calculated the spin-independent DM elastic scattering cross section on a nucleon. In fact, one should consider the uncertainties in the DM direct detection induced by the uncertainties in the hadronic matrix elements. Here we consider these uncertainties and recalculate the spin-independent DM-nucleon elastic scattering cross section. Then we calculate the neutrino fluxes from the singlet scalar DM annihilation in the Sun and the neutrino induced upgoing muon fluxes in the Super-Kamiokande and IceCube. This paper is organized as follows: In Sec. II, we outline the main features of the SSDM-SM and SSDM2HBDM, and give the DM-nucleon elastic scattering cross section. In Sec. III, we numerically calculate the differential neutrino energy spectrum generated by per DM pair annihilation, the DM annihilation rate in the Sun and the neutrino induced upgoing muon fluxes. Some discussions and conclusions are given in Sec. IV.

\section{CONSTRAINT ON SINGLET SCALAR DARK MATTER FROM DIRECT DETECTIONS}

\section{A. The real singlet scalar dark matter model as an extension of the SM}

In the SSDM-SM, the Lagrangian reads

$$
\mathcal{L}=\mathcal{L}_{\mathrm{SM}}+\frac{1}{2} \partial_{\mu} S \partial^{\mu} S-\frac{m_{0}^{2}}{2} S^{2}-\frac{\lambda_{S}}{4} S^{4}-\lambda S^{2} H^{\dagger} H
$$

where $H$ is the SM Higgs doublet. The linear and cubic terms of the scalar $S$ are forbidden by the $Z_{2}$ symmetry $S \rightarrow-S$. Then $S$ has a vanishing vacuum expectation value (VEV) $\langle S\rangle=0$ which ensures the DM candidate $S$ stable. $\lambda_{S}$ describes the DM self-interaction strength which is independent of the DM annihilation. After the spontaneous symmetry breaking (SSB), one can obtain the DM mass $m_{D}^{2}=m_{0}^{2}+\lambda v_{\mathrm{EW}}^{2}$ with $v_{\mathrm{EW}}=246 \mathrm{GeV}$. The SSDM-SM is very simple and has only three free parameters: the DM mass $m_{D}$, the Higgs mass $m_{h}$ and the coupling $\lambda$. In terms of the observed DM abundance $\Omega_{\mathrm{DM}} h^{2}=0.1123 \pm 0.0035$ [3], one can calculate the coupling $\lambda$ for the given $m_{D}$ and $m_{h}$. Here we take $m_{h}=125 \mathrm{GeV}$ [20] and $1 \mathrm{GeV} \leq m_{D} \leq 200 \mathrm{GeV}$ for illustration. As shown in Fig. 1, the observed DM abundance requires $\lambda \sim O\left(10^{-4}-1\right)$. It is well 
known that the annihilation cross section $\sigma$ will become larger for the same coupling when the annihilation process nears a resonance. This feature implies that there is a very small coupling when $0.8 m_{h} \lesssim 2 m_{D}<m_{h}$ as shown in Fig. 1. This region is named as the resonance region in the following parts of this paper.
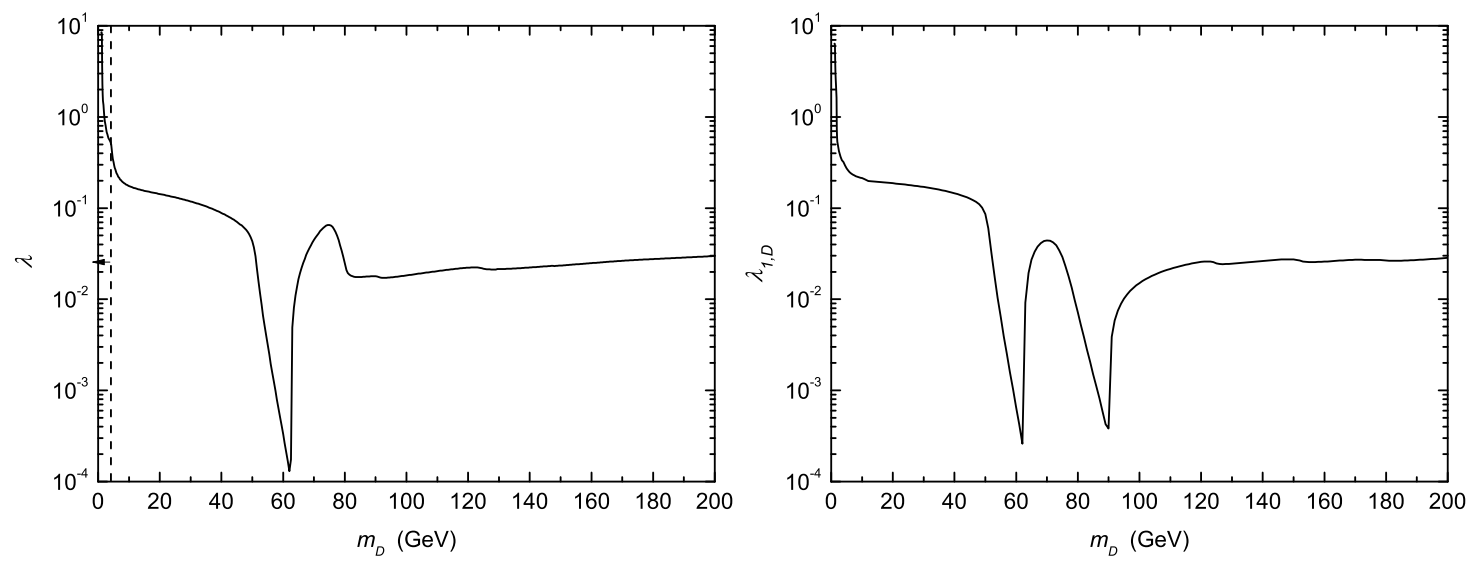

FIG. 1: The predicted couplings $\lambda$ in the SSDM-SM (left panel) and $\lambda_{1, D}$ in the SSDM-2HBDM (right panel) as a function of the DM mass $m_{D}$ from the observed DM abundance $\Omega_{\mathrm{DM}} h^{2}=0.1123 \pm 0.0035$ [3]. The vertical dashed line with arrowhead in the left panel shows the excluded region from the potential's global minimum, perturbativity and DM relic density.

Using the predicted $\lambda$ from the observed DM abundance, one can calculate the spin-independent DM-nucleon elastic scattering cross section [21]

$$
\sigma_{n}^{\mathrm{SI}} \approx \frac{\lambda^{2}}{\pi} f^{2} \frac{m_{n}^{2}}{m_{h}^{4} m_{D}^{2}}\left(\frac{m_{D} m_{n}}{m_{D}+m_{n}}\right)^{2},
$$

where $m_{n}$ is the nucleon mass and $f=(7 / 9) \sum_{q=u, d, s} f_{T q}^{p}+2 / 9$. In terms of the relevant formulas in Ref. [8], one can calculate the parameters $f_{T q}^{p}$ and obtain $f \approx 0.56 \pm 0.17$. On the other hand, the lattice results imply $f \approx 0.29 \pm 0.03$ where we take the strange-quark sigma term $16 \mathrm{MeV} \leq$ $\sigma_{s} \leq 69 \mathrm{MeV}$ [22]. Therefore we adopt $0.26 \leq f \leq 0.73$ for the following analyses. The authors in Ref. [15] have discussed that the light DM particle $S$ can explain the DAMA [23] and CoGeNT [24] experiments. Here we consider the latest experiment limits and recalculate the spin-independent DM-nucleon elastic scattering cross section $\sigma_{n}^{\mathrm{SI}}$ with $0.26 \leq f \leq 0.73$. Notice that $\sigma_{n}^{\mathrm{SI}}$ is not sensitive to the Higgs mass in the low DM mass range. As shown in Fig. 2 (top left panel), the predicted $\sigma_{n}^{\mathrm{SI}}$ in the region $6 \mathrm{GeV} \lesssim m_{D} \lesssim 8 \mathrm{GeV}$ and $f \gtrsim 0.60$ well fit the common region of the DAMA and CoGeNT [25]. However, the recent CDMS II [26] disfavors 
the CoGeNT+DAMA region. We find that the CDMS II [26], CDMS (shallow-site data) [27], CRESST [28] and TEXONO [29] can exclude the $f \gtrsim 0.63$ region for $1 \mathrm{GeV} \leq m_{D} \leq 10 \mathrm{GeV}$. The $f=0.63$ case has been shown as the blue solid line in the top left panel of Fig. 2, The latest XENON100 [30] may exclude $7 \mathrm{GeV} \lesssim m_{D} \lesssim 52 \mathrm{GeV}$ and a narrow region $65 \mathrm{GeV} \lesssim m_{D} \lesssim 80$ GeV even if we take $f=0.26$ as shown in Fig. 2. The future experiments CDMS $100 \mathrm{~kg}$ [31] and XENON1T [32] can cover most parts of the allowed parameter space. As the DM mass increases, new DM annihilation channels will be open which means that the predicted $\lambda$ from the DM relic density will quickly decrease. Therefore a kink around the bottom quark mass $m_{D} \approx m_{b}=4.2$ GeV occurs in the top left panel of Fig. 2. When the DM mass approaches the resonant point $m_{D}$ $=62.5 \mathrm{GeV}$ for $m_{h}=125 \mathrm{GeV}$, one can obtain a very large thermally averaged annihilation cross section $\langle\sigma v\rangle$ for the given $\lambda$. In order to derive the correct DM relic density, we have to require that the coupling $\lambda$ is very small. In this case, a very small $\sigma_{n}^{\text {SI }}$ around the resonant point can be obtained.

The SSDM-SM also suffers other constraints except for the direct detections, such as the potential's global minimum at $\langle h\rangle=v_{\mathrm{EW}}$ and $\langle S\rangle=0$ requires $|\lambda|<\sqrt{\lambda_{S} / 2} m_{h} / v_{\mathrm{EW}}+m_{D}^{2} / v_{\mathrm{EW}}^{2}[13]$. Since the perturbativity implies $6 \lambda_{S}<4 \pi$, one can derive $|\lambda|<\sqrt{\pi / 3} m_{h} / v_{\mathrm{EW}}+m_{D}^{2} / v_{\mathrm{EW}}^{2}$. Then we find the desired DM relic density can exclude $m_{D} \lesssim 4.1 \mathrm{GeV}$ for $m_{h}=125 \mathrm{GeV}$. The vertical dashed line with arrowhead in the left panels of Figs. 1 and 2 shows the excluded region. In Ref. [14], the authors have given the lower bounds on $m_{D}$ for several typical $\lambda_{S}$ based on the one-loop vacuum stability and the observed DM relic density.

\section{B. The complex singlet scalar dark matter model as an extension of the 2HBDM}

We begin with a brief review of the 2HBDM described in Ref. [17]. The model is based on the gauge group $S U(2)_{L} \otimes S U(2)_{R} \otimes U(1)_{B-L}$. The left- and right-handed fermions belong to $S U(2)_{L}$ and $S U(2)_{R}$ doublets, respectively. The Higgs sector contains two Higgs bidoublets $\phi\left(2,2^{*}, 0\right), \chi$ $\left(2,2^{*}, 0\right)$ and a left(right)-handed Higgs triplet $\Delta_{L(R)}(3(1), 1(3), 2)$ with the following flavor contents

$$
\phi=\left(\begin{array}{ll}
\phi_{1}^{0} & \phi_{2}^{+} \\
\phi_{1}^{-} & \phi_{2}^{0}
\end{array}\right), \chi=\left(\begin{array}{ll}
\chi_{1}^{0} & \chi_{2}^{+} \\
\chi_{1}^{-} & \chi_{2}^{0}
\end{array}\right), \Delta_{L, R}=\left(\begin{array}{cc}
\delta_{L, R}^{+} / \sqrt{2} & \delta_{L, R}^{++} \\
\delta_{L, R}^{0} & -\delta_{L, R}^{+} / \sqrt{2}
\end{array}\right) .
$$

The introduction of Higgs bidoublets $\phi$ and $\chi$ can account for the electroweak symmetry breaking and overcome the fine-tuning problem in generating the spontaneous $C P$ violation in the left-right 

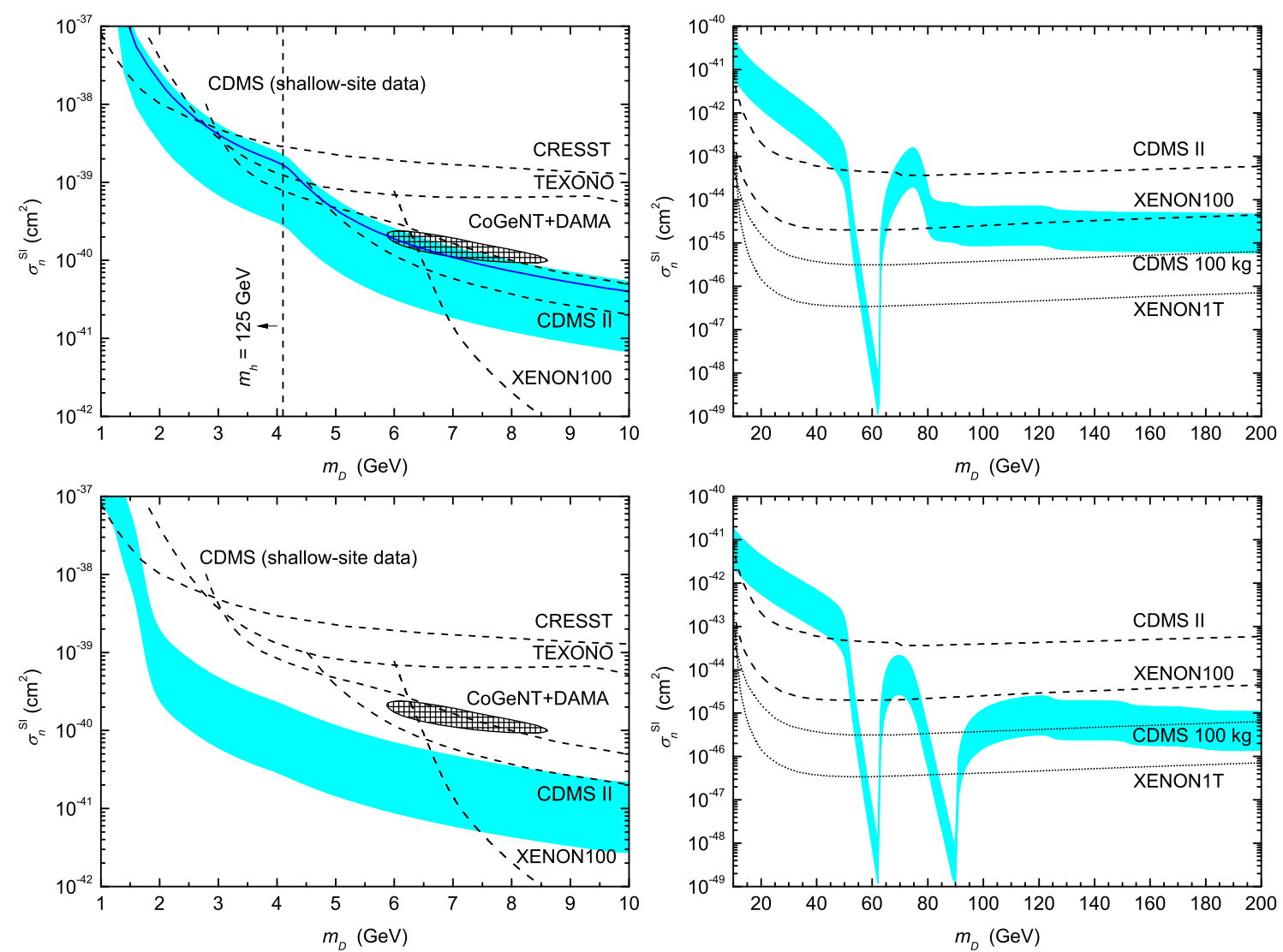

FIG. 2: The predicted DM-nucleon elastic scattering cross section $\sigma_{n}^{\text {SI }}$ in the SSDM-SM (top row) and SSDM-2HBDM (bottom row) for $1 \mathrm{GeV} \leq m_{D} \leq 200 \mathrm{GeV}$. The dashed lines indicate the current experimental upper bounds. The short dotted lines in the right panels denote the future experimental upper bounds from the CDMS $100 \mathrm{~kg}$ [31] and XENON1T [32]. The blue solid line in the top left panel describes the $f=0.63$ case. The black hatched region corresponds to a combination of the DAMA and CoGeNT [25]. The vertical dashed line with arrowhead in the top left panel shows the excluded region from the potential's global minimum, perturbativity and DM relic density.

symmetric one Higgs bidoublet model. Meanwhile it also relaxes the severe low energy phenomenological constraints [17]. Motivated by the spontaneous $P$ and $C P$ violations, we require $P$ and $C P$ invariance of the Lagrangian, which strongly restricts the structure of the Higgs potential. 
The most general potential containing only the $\phi$ and $\Delta_{L, R}$ fields is given by

$$
\begin{aligned}
\mathcal{V}_{\phi \Delta}= & -\mu_{1}^{2} \operatorname{Tr}\left(\phi^{\dagger} \phi\right)-\mu_{2}^{2}\left[\operatorname{Tr}\left(\tilde{\phi}^{\dagger} \phi\right)+\operatorname{Tr}\left(\tilde{\phi} \phi^{\dagger}\right)\right]-\mu_{3}^{2}\left[\operatorname{Tr}\left(\Delta_{\mathrm{L}} \Delta_{\mathrm{L}}^{\dagger}\right)+\operatorname{Tr}\left(\Delta_{\mathrm{R}} \Delta_{\mathrm{R}}^{\dagger}\right)\right] \\
& +\lambda_{1}\left[\operatorname{Tr}\left(\phi^{\dagger} \phi\right)\right]^{2}+\lambda_{2}\left\{\left[\operatorname{Tr}\left(\tilde{\phi}^{\dagger} \phi\right)\right]^{2}+\left[\operatorname{Tr}\left(\tilde{\phi} \phi^{\dagger}\right)\right]^{2}\right\}+\lambda_{3}\left[\operatorname{Tr}\left(\tilde{\phi}^{\dagger} \phi\right) \operatorname{Tr}\left(\tilde{\phi} \phi^{\dagger}\right)\right] \\
& +\lambda_{4}\left\{\operatorname{Tr}\left(\phi^{\dagger} \phi\right)\left[\operatorname{Tr}\left(\tilde{\phi}^{\dagger} \phi\right)+\operatorname{Tr}\left(\tilde{\phi} \phi^{\dagger}\right)\right]\right\} \\
& +\rho_{1}\left\{\left[\operatorname{Tr}\left(\Delta_{\mathrm{L}} \Delta_{\mathrm{L}}^{\dagger}\right)\right]^{2}+\left[\operatorname{Tr}\left(\Delta_{\mathrm{R}} \Delta_{\mathrm{R}}^{\dagger}\right)\right]^{2}\right\}+\rho_{2}\left[\operatorname{Tr}\left(\Delta_{\mathrm{L}} \Delta_{\mathrm{L}}\right) \operatorname{Tr}\left(\Delta_{\mathrm{L}}^{\dagger} \Delta_{\mathrm{L}}^{\dagger}\right)+\operatorname{Tr}\left(\Delta_{\mathrm{R}} \Delta_{\mathrm{R}}\right) \operatorname{Tr}\left(\Delta_{\mathrm{R}}^{\dagger} \Delta_{\mathrm{R}}^{\dagger}\right)\right] \\
& +\rho_{3}\left[\operatorname{Tr}\left(\Delta_{\mathrm{L}} \Delta_{\mathrm{L}}^{\dagger}\right) \operatorname{Tr}\left(\Delta_{\mathrm{R}} \Delta_{\mathrm{R}}^{\dagger}\right)\right]+\rho_{4}\left[\operatorname{Tr}\left(\Delta_{\mathrm{L}} \Delta_{\mathrm{L}}\right) \operatorname{Tr}\left(\Delta_{\mathrm{R}}^{\dagger} \Delta_{\mathrm{R}}^{\dagger}\right)+\operatorname{Tr}\left(\Delta_{\mathrm{L}}^{\dagger} \Delta_{\mathrm{L}}^{\dagger}\right) \operatorname{Tr}\left(\Delta_{\mathrm{R}} \Delta_{\mathrm{R}}\right)\right] \\
& +\alpha_{1} \operatorname{Tr}\left(\phi^{\dagger} \phi\right)\left[\operatorname{Tr}\left(\Delta_{\mathrm{L}} \Delta_{\mathrm{L}}^{\dagger}\right)+\operatorname{Tr}\left(\Delta_{\mathrm{R}} \Delta_{\mathrm{R}}^{\dagger}\right)\right]+\alpha_{2} \operatorname{Tr}\left[\left(\tilde{\phi}^{\dagger} \phi\right)+\left(\tilde{\phi} \phi^{\dagger}\right)\right] \operatorname{Tr}\left[\left(\Delta_{\mathrm{L}} \Delta_{\mathrm{L}}^{\dagger}\right)+\left(\Delta_{\mathrm{R}} \Delta_{\mathrm{R}}^{\dagger}\right)\right] \\
& +\alpha_{3}\left[\operatorname{Tr}\left(\phi \phi^{\dagger} \Delta_{\mathrm{L}} \Delta_{\mathrm{L}}^{\dagger}\right)+\operatorname{Tr}\left(\phi^{\dagger} \phi \Delta_{\mathrm{R}} \Delta_{\mathrm{R}}^{\dagger}\right)\right] \\
& +\beta_{1}\left[\operatorname{Tr}\left(\phi \Delta_{\mathrm{R}} \phi^{\dagger} \Delta_{\mathrm{L}}^{\dagger}\right)+\operatorname{Tr}\left(\phi^{\dagger} \Delta_{\mathrm{L}} \phi \Delta_{\mathrm{R}}^{\dagger}\right)\right]+\beta_{2}\left[\operatorname{Tr}\left(\tilde{\phi} \Delta_{\mathrm{R}} \phi^{\dagger} \Delta_{\mathrm{L}}^{\dagger}\right)+\operatorname{Tr}\left(\tilde{\phi}^{\dagger} \Delta_{\mathrm{L}} \phi \Delta_{\mathrm{R}}^{\dagger}\right)\right] \\
& +\beta_{3}\left[\operatorname{Tr}\left(\phi \Delta_{\mathrm{R}} \tilde{\phi}^{\dagger} \Delta_{\mathrm{L}}^{\dagger}\right)+\operatorname{Tr}\left(\phi^{\dagger} \Delta_{\mathrm{L}} \tilde{\phi} \Delta_{\mathrm{R}}^{\dagger}\right)\right],
\end{aligned}
$$

where the coefficients $\mu_{i}, \lambda_{i}, \rho_{i}, \alpha_{i}$ and $\beta_{i}$ in the potential are all real as all the terms are selfHermitian. The Higgs potential $\mathcal{V}_{\chi \Delta}$ involving $\chi$ field can be obtained by the replacement $\chi \leftrightarrow \phi$ in Eq. (4). The mixing term $\mathcal{V}_{\chi \phi \Delta}$ can be obtained by replacing one of $\phi$ by $\chi$ in all the possible ways in Eq. (4). After the SSB, the Higgs multiplets obtain nonzero VEVs

$$
\left\langle\phi_{1,2}^{0}\right\rangle=\frac{\kappa_{1,2}}{\sqrt{2}},\left\langle\chi_{1,2}^{0}\right\rangle=\frac{w_{1,2}}{\sqrt{2}} \text { and }\left\langle\delta_{L, R}^{0}\right\rangle=\frac{v_{L, R}}{\sqrt{2}} \text {, }
$$

where $\kappa_{1}, \kappa_{2}, w_{1}, w_{2}, v_{L}$ and $v_{R}$ are in general complex, and $\kappa \equiv \sqrt{\left|\kappa_{1}\right|^{2}+\left|\kappa_{2}\right|^{2}+\left|w_{1}\right|^{2}+\left|w_{2}\right|^{2}} \approx 246$ $\mathrm{GeV}$ represents the electroweak symmetry breaking scale. The value of $v_{R}$ sets the scale of leftright symmetry breaking which is directly linked to the right-handed gauge boson masses. $v_{R}$ is subjected to strong constraints from the $K, B$ meson mixing as well as low energy electroweak interactions. The kaon mass difference and the indirect $C P$ violation quantity $\epsilon_{K}$ set a bound for $v_{R}$ around $10 \mathrm{TeV}$ [33]. In general, the $2 \mathrm{HBDM}$ includes three light neutral Higgs bosons and a pair of charged light Higgs particles, whose masses are order of the electroweak energy scale. For simplicity, we consider $\kappa_{2} \sim w_{2} \sim 0$. Then one can derive three light neutral Higgs bosons: $h, H, A$ from $\phi_{1}^{0}$ and $\chi_{1}^{0}$, and a pair of charged light Higgs particles: $h^{ \pm}$from $\chi_{1}^{ \pm}$. For a concrete numerical illustration, we choose all the masses $m_{H}, m_{A}, m_{h^{ \pm}}=180 \mathrm{GeV}$ and $m_{h}=125 \mathrm{GeV}$.

In the $2 \mathrm{HBDM}$, the $P$ and $C P$ symmetries have been required to be exactly conserved before the SSB, thus the discrete symmetries $P$ and $C P$ can be used to stabilize the DM candidate. In the framework of 2HBDM with a complex singlet scalar $S=\left(S_{\sigma}+i S_{D}\right) / \sqrt{2}(\mathrm{SSDM}-2 \mathrm{HBDM})$, we have considered this possibility in Ref. [19]. The $P$ and $C P$ transformation properties of the 


\begin{tabular}{|c|c|c||c|c|c||c|c|c|}
\hline & $P$ & $C P$ & & $P$ & $C P$ & & $P$ & $C P$ \\
\hline$S$ & $S$ & $S^{*}$ & $S+S^{*}$ & + & + & $S-S^{*}$ & + & - \\
\hline$\phi$ & $\phi^{\dagger}$ & $\phi^{*}$ & $S S^{*}$ & + & + & $\operatorname{Tr}\left(\phi^{\dagger} \phi\right)$ & + & + \\
\hline$\tilde{\phi}$ & $\tilde{\phi}^{\dagger}$ & $\tilde{\phi}^{*}$ & $\operatorname{Tr}\left(\phi^{\dagger} \tilde{\phi} \pm \tilde{\phi}^{\dagger} \phi\right)$ & \pm & \pm & $\operatorname{Tr}\left(\chi^{\dagger} \tilde{\chi} \pm \tilde{\chi}^{\dagger} \chi\right)$ & \pm & \pm \\
\hline$\chi$ & $\chi^{\dagger}$ & $\chi^{*}$ & $\operatorname{Tr}\left(\chi^{\dagger} \tilde{\phi} \pm \tilde{\phi}^{\dagger} \chi\right)$ & \pm & \pm & $\operatorname{Tr}\left(\phi^{\dagger} \chi \pm \chi^{\dagger} \phi\right)$ & \pm & \pm \\
\hline$\Delta_{L(R)}$ & $\Delta_{R(L)}$ & $\Delta_{L(R)}^{*}$ & $\operatorname{Tr}\left(\Delta_{L}^{\dagger} \Delta_{L}+\Delta_{R}^{\dagger} \Delta_{R}\right)$ & + & + & $\operatorname{Tr}\left(\Delta_{L}^{\dagger} \Delta_{L}-\Delta_{R}^{\dagger} \Delta_{R}\right)$ & - & + \\
\hline
\end{tabular}

TABLE I: The $P$ and $C P$ transformation properties of the Higgs particles and their gauge-invariant combinations. The "+" and "-" denote even and odd, respectively.

Higgs particles and their gauge-invariant combinations have been shown in Table I. It is clear that the odd powers of $\left(S-S^{*}\right)$ are forbidden by the $P$ and $C P$ symmetries. This hidden discrete $Z_{2}$ symmetry on $S_{D}$ is induced from the original $P$ and $C P$ symmetries. With the help of this hidden $Z_{2}$ symmetry, one may derive $\left\langle S_{D}\right\rangle=0$ or $\left\langle S_{D}\right\rangle \neq 0$ for the VEV of $S_{D}$. Since the $\left\langle S_{D}\right\rangle \neq 0$ case means that $S_{D}$ may decay and can not be the DM candidate, we require that $S$ obtains a real $\operatorname{VEV}\langle S\rangle=v_{\sigma} / \sqrt{2}$. Although both $P$ and $C P$ are broken after the SSB, there is still a residual $Z_{2}$ symmetry on $S_{D}$. Therefore $S_{D}$ is a stable particle and can be the DM candidate. We have checked that the $P$ and $C P$ transformation rules for $S$ defined in Table $\prod$ is actually the only possible way for the implementation of the DM candidate.

For the annihilation cross section of approximately weak strength, we expect that the DM mass is in the range of a few $\mathrm{GeV}$ and a few hundred $\mathrm{GeV}$. However, the mass $m_{D}$ of $S_{D}$ is related to the LR symmetry breaking scale $v_{R} \sim 10 \mathrm{TeV}$. To have a possible light DM mass, we may consider an approximate global $U(1)$ symmetry on $S$, i.e. $S \rightarrow e^{i \delta} S$. Then the $P$ and $C P$ invariant Higgs potential involving the singlet $S$ is given by

$$
\mathcal{V}_{S}=-\mu_{D}^{2} S S^{*}+\lambda_{D}\left(S S^{*}\right)^{2}+\sum_{i=1}^{7} \lambda_{i, D} S S^{*} O_{i}-\frac{m_{D}^{2}}{4}\left(S-S^{*}\right)^{2}
$$

where $O_{1}=\operatorname{Tr}\left(\phi^{\dagger} \phi\right), O_{2}=\operatorname{Tr}\left(\phi^{\dagger} \tilde{\phi}+\tilde{\phi}^{\dagger} \phi\right), O_{3}=\operatorname{Tr}\left(\chi^{\dagger} \chi\right), O_{4}=\operatorname{Tr}\left(\chi^{\dagger} \tilde{\chi}+\tilde{\chi}^{\dagger} \chi\right), O_{5}=\operatorname{Tr}\left(\phi^{\dagger} \chi+\chi^{\dagger} \phi\right)$, $O_{6}=\operatorname{Tr}\left(\chi^{\dagger} \tilde{\phi}+\tilde{\phi}^{\dagger} \chi\right)$ and $O_{7}=\operatorname{Tr}\left(\Delta_{L}^{\dagger} \Delta_{L}+\Delta_{R}^{\dagger} \Delta_{R}\right)$. Only the last term explicitly violates $U(1)$ symmetry. After the SSB, $S$ obtains a real $\operatorname{VEV} v_{\sigma} / \sqrt{2}$. Then one can straightly derive

$$
\mathcal{V}_{S}=\frac{\lambda_{D}}{4}\left[\left(S_{\sigma}^{2}+2 v_{\sigma} S_{\sigma}+S_{D}^{2}\right)^{2}-v_{\sigma}^{4}\right]+\sum_{i=1}^{7} \frac{\lambda_{i, D}}{2}\left(S_{\sigma}^{2}+2 v_{\sigma} S_{\sigma}+v_{\sigma}^{2}+S_{D}^{2}\right)\left(O_{i}-\left\langle O_{i}\right\rangle\right)+\frac{m_{D}^{2}}{2} S_{D}^{2}
$$

where we have used the minimization condition $\mu_{D}^{2}=\lambda_{D} v_{\sigma}^{2}+\sum_{i} \lambda_{i, D}\left\langle O_{i}\right\rangle$ from the singlet $S_{\sigma}$ to 
eliminate the parameter $\mu_{D}$. The terms proportional to odd powers of $S_{D}$ are absent in Eq. (7) which implies $S_{D}$ can only be produced by pairs. Notice that the mass term of $S_{D}$ should be absent with an exact global $U(1)$ symmetry. As discussed in Ref. [19], the explicit breaking of this $U(1)$ symmetry can explain the naturalness of a light DM mass $m_{D}$, but it does not destroy the stability of the DM candidate $S_{D}$. For the VEV of $S_{\sigma}$, we require $v_{\sigma}>v_{R} \sim 10 \mathrm{TeV} \gg \kappa$ which means the mixing angles between $S_{\sigma}$ and other neutral Higgs bosons in the SSDM-2HBDM are small and the mass of $S_{\sigma}$ is very heavy.

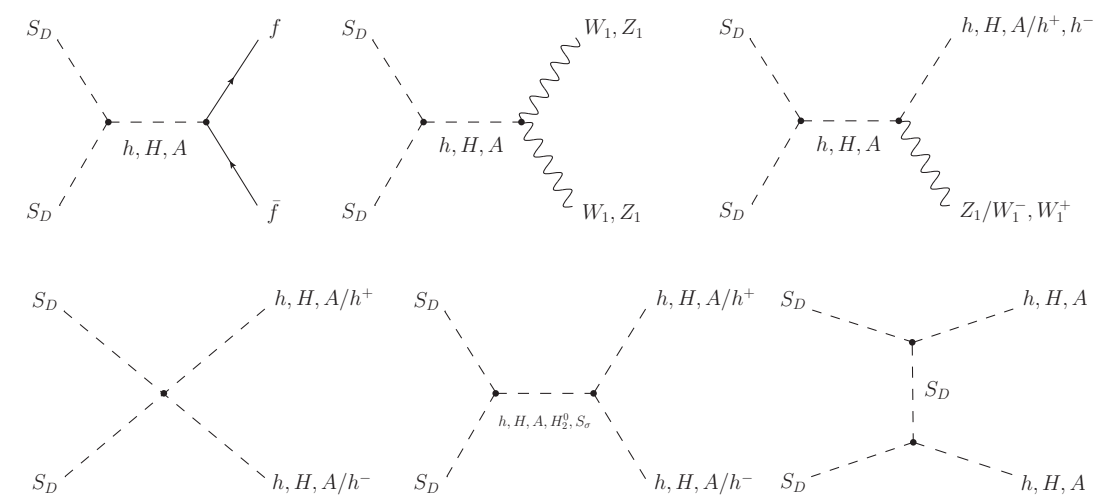

FIG. 3: Feynman diagrams for the DM annihilation in the SSDM-2HBDM.

For the DM mass, we take $1 \mathrm{GeV} \leq m_{D} \leq 200 \mathrm{GeV}$. In this case, the possible DM annihilation products are $f \bar{f}, W_{1} W_{1} / Z_{1} Z_{1}, W_{1}^{ \pm} h^{\mp} / Z_{1}(h, H, A), h^{+} h^{-}$and any two of the three neutral states $(h, H, A)$ as shown in Fig. 3. Here $W_{1}$ and $Z_{1}$ denote the SM gauge bosons. For cubic and quartic scalar vertexes, we assume they are the same as those in the one Higgs bidoublet case [19]. Namely, the vertexes of $S_{D} S_{D}(h, H, A)$ and $S_{D} S_{D}\left(h, H, A / h^{+}\right)\left(h, H, A / h^{-}\right)$are set equal to $-i \lambda_{1, D} v_{\mathrm{EW}}$ and $-i \lambda_{1, D}$, respectively. Similarly, the cubic scalar vertexes among the light Higgs particles $h, H, A$ and $h^{ \pm}$are set equal to $-i 3 m_{h}^{2} / \nu_{\mathrm{EW}}$, and the cubic scalar vertexes between $S_{\sigma}$ and two light Higgs particles are assumed to be $-i \lambda_{1, D} v_{\sigma}$. The vertexes of $f \bar{f}(h, H, A)$ are related with the light Higgs mixing and the Yukawa scale factors $R_{q} . R_{q}$ controls the Yukawa couplings and its definition can be found in Eq. (28) of Ref. [19]. Once the light Higgs mixing and $R_{q}$ are fixed, one can predict the coupling $\lambda_{1, D}$ from the DM relic density.

In the SSDM-2HBDM, the DM-nucleon elastic scattering cross section is given by [19]

$$
\sigma_{n}^{\mathrm{SI}} \approx \frac{\lambda_{1, D}^{2}}{4 \pi} f^{2} \frac{m_{n}^{2}}{m_{D}^{2}}\left(\frac{m_{D} m_{n}}{m_{D}+m_{n}}\right)^{2}\left(\frac{f_{1}}{m_{h}^{2}}+\frac{f_{3}}{m_{H}^{2}}+\frac{f_{5}}{m_{A}^{2}}\right)^{2} .
$$


The parameters $f_{1}, f_{3}$ and $f_{5}$ have been given in Ref. [19] and are related with the light Higgs mixing and the Yukawa scale factors $R_{q}$. Neglecting possible cancelation due to the light Higgs mixing in Eq. (8), we find that $\sigma_{n}^{\mathrm{SI}}$ can be enhanced by the large $R_{q}$ and approach the current experimental upper bound for the heavy DM mass [19]. On the other hand, one can also adjust $R_{q}$ to avoid the current direct detection limits for the light DM mass. For illustration, we take the Yukawa scale factors $R_{q}=1$ for quarks and $R_{l}=10$ for charged leptons. Meanwhile, we consider the case II for the light Higgs mixing [19]. In terms of the observed DM relic density, we calculate the allowed coupling $\lambda_{1, D}$ for $1 \mathrm{GeV} \leq m_{D} \leq 200 \mathrm{GeV}$. As shown in Fig. 11 (right panel), the observed DM abundance requires $\lambda \sim O\left(10^{-4}-1\right)$. Then we plot the predicted $\sigma_{n}^{\text {SI }}$ with $0.26 \leq f \leq 0.73$ in Fig. 2 (bottom row). The latest XENON100 [30] may exclude $7.5 \mathrm{GeV} \lesssim$ $m_{D} \lesssim 52 \mathrm{GeV}$ and $67 \mathrm{GeV} \lesssim m_{D} \lesssim 72 \mathrm{GeV}$. It is clear that the SSDM-2HBDM has smaller $\sigma_{n}^{\text {SI }}$ than that in the SSDM-SM for $1 \mathrm{GeV} \leq m_{D} \leq 10 \mathrm{GeV}$. In the next section, we shall see another advantage of the SSDM-2HBDM. Namely, the SSDM-2HBDM can give larger neutrino induced upgoing muon fluxes than those in the SSDM-SM even if the SSDM-2HBDM has the smaller $\sigma_{n}^{\mathrm{SI}}$. This is because that the large $R_{l}$ can significantly change the branching ratios of the dominant DM annihilation channels which are relevant to the produced neutrino fluxes.

\section{NEUTRINO SIGNALS FROM THE DARK MATTER ANNIHILATION IN THE SUN}

Based on the DM mass $m_{D}$ discussed in this paper, two DM particles may annihilate into fermion pairs, gauge boson pairs and Higgs pairs. Therefore the differential muon neutrino energy spectrum at the surface of the Earth from per DM pair annihilation in the Sun can be written as

$$
\frac{d N_{v_{\mu}}}{d E_{v_{\mu}}}=\sum_{f s} B_{f s} \frac{d N_{v_{\mu}}^{f s}}{d E_{v_{\mu}}},
$$

where $f s$ denotes the DM annihilation final state and $B_{f_{s}}$ is the branching ratio into the final state $f s$. $B_{f s}$ can be exactly calculated when the couplings $\lambda$ and $\lambda_{1, D}$ are obtained from the DM relic density. $d N_{v_{\mu}}^{f s} / d E_{v_{\mu}}$ is the energy distribution of neutrinos at the surface of the Earth produced by the final state $f s$ through hadronization and decay processes in the core of the Sun. It should be mentioned that some produced particles, such as $B$ mesons and muons, can lose a part of energy or the total energy before they decay due to their interactions in the Sun. In addition, we should consider the neutrino interactions in the Sun and neutrino oscillations. In this paper, we use the program package WimpSim [34] to calculate $d N_{v_{\mu}}^{f s} / d E_{v_{\mu}}$ with the help of Pythia [35], Nusigma 
[36] and DarkSUSY [37]. The Pythia can help us to simulate the hadronization and decay of the annihilation products and collect the produced neutrinos and antineutrinos. The Nusigma is a neutrino-nucleon scattering Monte Carlo package for neutrino interactions on the way out of the Sun. The density profile of the Sun may affect neutrino oscillations due to matter effects. For the solar density, the WimpSim uses the standard solar model BS05(OP) [38] which is coded into the DarkSUSY. Notice that the WimpSim does not simulate the Higgs annihilation channel. Since the Higgs decay branching ratios and the energy distribution of the Higgs decay products can be exactly calculated in the SSDM-SM, the differential neutrino energy spectrum from the Higgs annihilation channel can be generated by those from other annihilation channels. Except for the DM masses and annihilation channels, the WimpSim only requires inputs of the neutrino oscillation parameters. Here we consider the lastest Daya Bay results [39] and take [40]

$$
\begin{gathered}
\sin ^{2} \theta_{12}=0.32, \quad \sin ^{2} \theta_{23}=0.49, \quad \sin ^{2} \theta_{13}=0.026, \quad \delta=0.83 \pi, \\
\Delta m_{21}^{2}=7.62 \times 10^{-5} \mathrm{eV}^{2}, \quad \Delta m_{31}^{2}=2.53 \times 10^{-3} \mathrm{eV}^{2},
\end{gathered}
$$

for the neutrino oscillation parameters. Once $d N_{v_{\mu}} / d E_{v_{\mu}}$ is obtained, we can use the following equation to calculate the differential muon neutrino flux from the solar DM annihilation:

$$
\frac{d \Phi_{v_{\mu}}}{d E_{v_{\mu}}}=\frac{\Gamma_{\mathrm{ANN}}}{4 \pi R_{\mathrm{ES}}^{2}} \frac{d N_{v_{\mu}}}{d E_{v_{\mu}}}
$$

where $R_{\mathrm{ES}}=1.496 \times 10^{13} \mathrm{~cm}$ is the Earth-Sun distance. The solar DM annihilation rate $\Gamma_{\mathrm{ANN}}$ will be given in Eq. (20). In addition, we should also calculate the differential muon anti-neutrino flux which can be evaluated by an equation similar to Eq. (11).

\section{A. Dark matter capture rate and annihilation rate in the Sun}

The halo DM particles can be captured by the Sun via elastic scattering off solar nuclei. On the other hand, the DM annihilation in the Sun depletes the DM population. The evolution of the DM number $N$ in the Sun is given by the following equation [41]:

$$
\dot{N}=C_{\odot}-C_{E} N-C_{A} N^{2}
$$

where the dot denotes differentiation with respect to time. The solar capture rate $C_{\odot}$ may be approximately written as [1]

$$
C_{\odot} \approx 4.8 \times 10^{24} \mathrm{~s}^{-1} \frac{\rho_{\mathrm{DM}}}{0.3 \mathrm{GeV} / \mathrm{cm}^{3}} \frac{270 \mathrm{~km} / \mathrm{s}}{\bar{v}} \frac{1 \mathrm{GeV}}{m_{D}} \sum_{i} F_{i}\left(m_{D}\right) \frac{\sigma_{\mathrm{N}_{i}}^{\mathrm{SI}}}{10^{-40} \mathrm{~cm}^{2}} f_{i} \phi_{i} S\left(\frac{m_{D}}{m_{\mathrm{N}_{i}}}\right) \frac{1 \mathrm{GeV}}{m_{\mathrm{N}_{i}}}(13)
$$


where $\sigma_{\mathrm{N}_{i}}^{\mathrm{SI}}$ is the spin-independent cross section of the DM elastic scattering off nucleus $\mathrm{N}_{i}$. For the local DM density $\rho_{\mathrm{DM}}$ and the local DM root-mean-square velocity $\bar{v}$, we take $\rho_{\mathrm{DM}}=0.3 \mathrm{GeV} / \mathrm{cm}^{3}$ and $\bar{v}=270 \mathrm{~km} / \mathrm{s} . f_{i}$ and $\phi_{i}$ describe the mass fraction and the distribution of the element $i$ in the Sun, respectively. $f_{i}, \phi_{i}$ and the form-factor suppression $F_{i}\left(m_{D}\right)$ can be found in Ref. [1]. The function $S(x)$ denotes the kinematic suppression and is given by

$$
S(x)=\left[\frac{A(x)^{1.5}}{1+A(x)^{1.5}}\right]^{2 / 3}
$$

with

$$
A(x)=\frac{3 x}{2(x-1)^{2}}\left(\frac{\left\langle v_{\mathrm{esc}}\right\rangle}{\bar{v}}\right)^{2}
$$

where $\left\langle v_{\mathrm{esc}}\right\rangle=1156 \mathrm{~km} \mathrm{~s}^{-1}$ is a mean escape velocity. In Eq. (12), the term $C_{E} N$ describes the DM evaporation rate. For the parameter $C_{E}$, we adopt the following approximate formula [6, 42]

$$
C_{E} \approx 10^{-3.5\left(m_{D} / \mathrm{GeV}\right)-4} \mathrm{~s}^{-1} \frac{\sigma_{\mathrm{H}}^{\mathrm{SI}}}{5 \times 10^{-39} \mathrm{~cm}^{2}} .
$$

The last term $C_{A} N^{2}$ in Eq. (12) controls the DM annihilation rate in the Sun. The coefficient $C_{A}$ depends on the thermal-average of the annihilation cross section times the relative velocity $\langle\sigma v\rangle$ and the DM distribution in the Sun. To a good approximation,

$$
C_{A}=\frac{\langle\sigma v\rangle}{V_{\mathrm{eff}}}
$$

where $V_{\text {eff }}$ is the effective volume of the core of the Sun and is given by [41]

$$
V_{\mathrm{eff}}=5.8 \times 10^{30} \mathrm{~cm}^{3}\left(\frac{1 \mathrm{GeV}}{m_{D}}\right)^{3 / 2} .
$$

It is worthwhile to stress that $\langle\sigma v\rangle$ in Eq. (17) should be evaluated at the solar central temperature $T_{c}=1.4 \times 10^{7} \mathrm{~K}$.

In Refs. [16] and [19], we have calculated the DM-nucleon elastic scattering cross section $\sigma_{n}^{\text {SI }}$ which is equal to $\sigma_{\mathrm{H}}^{\mathrm{SI}}$. The relation between $\sigma_{\mathrm{N}_{i}}^{\mathrm{SI}}$ and $\sigma_{\mathrm{H}}^{\mathrm{SI}}$ can be written as

$$
\sigma_{\mathrm{N}_{i}}^{\mathrm{SI}}=A_{\mathrm{N}_{i}}^{2} \frac{M^{2}\left(\mathrm{~N}_{i}\right)}{M^{2}(\mathrm{H})} \sigma_{\mathrm{H}}^{\mathrm{SI}}
$$

where $A_{\mathrm{N}_{i}}$ is the mass number of the nucleus $\mathrm{N}_{i}$ and $M(x)=m_{D} m_{x} /\left(m_{D}+m_{x}\right)$. If $m_{D} \gg m_{\mathrm{N}_{i}}$, we can easily derive $\sigma_{\mathrm{N}_{i}}^{\mathrm{SI}} \approx A_{\mathrm{N}_{i}}^{4} \sigma_{\mathrm{H}}^{\mathrm{SI}}$. Then one may find that the solar capture rate by other elements in the Sun is much larger than that by the hydrogen element although it has the maximal mass 
fraction. In terms of relevant formulas in Refs. [16] and [19], we calculate $\langle\sigma v\rangle$ at $T_{c}=1.4 \times 10^{7}$ $\mathrm{K}$. Using $\sigma_{\mathrm{H}}^{\mathrm{SI}}$ and $\langle\sigma v\rangle$, one can straightly calculate $C_{\odot}, C_{E}$ and $C_{A}$. Then we solve the evolution equation and derive the solar DM annihilation rate [41]

$$
\Gamma_{\mathrm{ANN}}=\frac{1}{2} C_{A} N^{2}=\frac{1}{2} C_{\odot}\left[\frac{\tanh \left(\kappa t_{\odot} \sqrt{C_{\odot} C_{A}}\right)}{\kappa+C_{E} /\left(2 \sqrt{C_{\odot} C_{A}}\right) \tanh \left(\kappa t_{\odot} \sqrt{C_{\odot} C_{A}}\right)}\right]^{2},
$$

where $\kappa=\sqrt{1+C_{E}^{2} /\left(4 C_{\odot} C_{A}\right)}$ and $t_{\odot} \simeq 4.5 \mathrm{Gyr}$ is the age of the solar system. When $C_{E}$ is small enough $\left(m_{D} \gtrsim 4 \mathrm{GeV}\right)$, one may neglect the evaporation effect and obtain

$$
\Gamma_{\mathrm{ANN}}=\frac{1}{2} C_{\odot} \tanh ^{2}\left(t_{\odot} \sqrt{C_{\odot} C_{A}}\right) .
$$

If $t_{\odot} \sqrt{C_{\odot} C_{A}} \gg 1$, the $\mathrm{DM}$ annihilation rate reaches equilibrium with the DM capture rate. In this case, we derive the maximal DM annihilation rate $\Gamma_{\mathrm{ANN}}=C_{\odot} / 2$ which is entirely determined by $C_{\odot}$. Therefore the enhanced $\langle\sigma v\rangle$ via the Breit-Wigner resonance enhancement mechanism [43] can not affect $\Gamma_{\mathrm{ANN}}$. For $m_{D} \gtrsim 4 \mathrm{GeV}$, we find that most parts of the parameter space reach or approach the equilibrium except for the resonance region. It is because that both $\sigma_{n}^{\mathrm{SI}}$ and $\langle\sigma v\rangle$ are very small in this region [16].

\section{B. Neutrino induced upgoing muon fluxes in the Super-Kamiokande}

The high energy muon neutrinos from the solar DM annihilation interact with the Earth rock to produce the upgoing muon flux which can be detected by the SK detector [10]. The neutrino induced muon flux is give by [44]

$$
\begin{aligned}
\Phi_{\mu}= & \int_{E_{\mathrm{thr}}^{\mathrm{SK}}}^{m_{D}} d E_{\mu} \int_{E_{\mu}}^{m_{D}} d E_{v_{\mu}} \frac{d \Phi_{v_{\mu}}}{d E_{v_{\mu}}} \int_{0}^{\infty} d L \int_{E_{\mu}}^{E_{v_{\mu}}} d E_{\mu}^{\prime} g\left(L, E_{\mu}, E_{\mu}^{\prime}\right) \sum_{a=p, n} \frac{d \sigma_{v_{\mu}}^{a}\left(E_{v_{\mu}}, E_{\mu}^{\prime}\right)}{d E_{\mu}^{\prime}} \rho_{a} \\
& +\left(v_{\mu} \rightarrow \bar{v}_{\mu}\right),
\end{aligned}
$$

where $\rho_{p} \approx 1 / 2 N_{A} \rho$ and $\rho_{n} \approx 1 / 2 N_{A} \rho$ are the number densities of protons and neutrons near the detector, respectively. $N_{A}$ is the Avogadro's number and $\rho$ is the density of the rock under the detector. $E_{\mathrm{thr}}^{\mathrm{SK}}=1.6 \mathrm{GeV}$ is the threshold energy of the SK detector. $g\left(L, E_{\mu}, E_{\mu}^{\prime}\right) d E_{\mu}$ is the probability that a muon of initial energy $E_{\mu}^{\prime}$ has energy between $E_{\mu}$ and $E_{\mu}+d E_{\mu}$ after propagating a distance $L$ in the rock. For the charged-current interaction cross sections, we use [4]

$$
\frac{d \sigma_{x}^{a}\left(E_{x}, E_{\mu}^{\prime}\right)}{d E_{\mu}^{\prime}} \approx \frac{2 m_{p} G_{F}^{2}}{\pi}\left(A_{x}^{a}+B_{x}^{a} \frac{E_{\mu}^{\prime 2}}{E_{x}^{2}}\right)
$$


where $A_{v_{\mu}}^{n, p}=0.25,0.15, B_{v_{\mu}}^{n, p}=0.06,0.04$ and $A_{\bar{v}_{\mu}}^{n, p}=B_{v_{\mu}}^{p, n}, B_{\bar{v}_{\mu}}^{n, p}=A_{v_{\mu}}^{p, n}$. The probability $g\left(L, E_{\mu}, E_{\mu}^{\prime}\right)$ can be obtained from the full Monte Carlo calculation of muon propagation. Here we use the approximation formula [44]

$$
g\left(L, E_{\mu}, E_{\mu}^{\prime}\right)=\frac{\delta\left(L-L_{0}\right)}{\rho\left(\alpha+\beta E_{\mu}\right)},
$$

with

$$
L_{0}=\frac{1}{\rho \beta} \ln \frac{\alpha+\beta E_{\mu}^{\prime}}{\alpha+\beta E_{\mu}},
$$

where $\alpha=2.3 \times 10^{-3} \mathrm{~g}^{-1} \mathrm{GeV} \mathrm{cm}{ }^{2}$ and $\beta=4.4 \times 10^{-6} \mathrm{~g}^{-1} \mathrm{~cm}^{2}$ describe muon energy loss in the standard rock [45]. It is shown that this analytic approximation is good to within $10 \%$ or better [44]. Then one can derive

$$
\Phi_{\mu}=\int_{E_{\mathrm{thr}}^{\mathrm{SK}}}^{m_{D}} d E_{\mu} \frac{1}{\rho\left(\alpha+\beta E_{\mu}\right)} \int_{E_{\mu}}^{m_{D}} d E_{v_{\mu}} \frac{d \Phi_{v_{\mu}}}{d E_{v_{\mu}}} \int_{E_{\mu}}^{E_{v_{\mu}}} d E_{\mu}^{\prime} \sum_{a=p, n} \frac{d \sigma_{v_{\mu}}^{a}\left(E_{v_{\mu}}, E_{\mu}^{\prime}\right)}{d E_{\mu}^{\prime}} \rho_{a}+\left(v_{\mu} \rightarrow \bar{v}_{\mu}\right) .
$$

Using a change of variable, we find that the formula in Eq. (26) is consistent with that in Ref. [7].

For the SSDM-SM, we calculate the neutrino induced upgoing muon fluxes in the SuperKamiokande with the help of Eqs. (11), (20) and (26). The numerical results have been shown in Fig. 4 (top row). Due to the multiple Coulomb scattering of muons on route to the detector, the final directions of muons are spread. For $10 \mathrm{GeV} \leq m_{D} \leq 200 \mathrm{GeV}$, the cone half-angles range from $5^{\circ}$ to $25^{\circ}$ [46]. Therefore we conservatively take $\Phi_{\mu} \leq 1.6 \times 10^{-14} \mathrm{~cm}^{-2} \mathrm{~s}^{-1}$ (maximal value in Fig. 8 of Ref. [10]) for the Super-Kamiokande limit. It is clear that our results in the region $3.7 \mathrm{GeV} \leq m_{D} \leq 4.2 \mathrm{GeV}$ and $f \gtrsim 0.65$ slightly exceed the Super-Kamiokande limit. Since the uncertainties in the astrophysics and particle physics, such as $\rho_{\mathrm{DM}}, \bar{v}$ and $\alpha$, we can not claim that the Super-Kamiokande can exclude this region. Notice that the exceeded region is not consistent with the CDMS (shallow-site data) results as shown in Fig. 2 (top left panel). For $10 \mathrm{GeV} \leq m_{D} \leq 200$ $\mathrm{GeV}$, our numerical results in Fig. 4 (top right panel) show that the predicted muon fluxes are less than the Super-Kamiokande limit. In Fig. 11 of Ref. [10], the Super-Kamiokande collaboration has also given the neutrino induced upgoing muon flux limits as a function of the DM mass. Their simulations assume that $80 \%$ of the annihilation products are from $b \bar{b}, 10 \%$ from $c \bar{c}$ and $10 \%$ from $\tau^{+} \tau^{-}$. It is found that $\Phi_{\mu} \leq 6.4 \times 10^{-15} \mathrm{~cm}^{-2} \mathrm{~s}^{-1}$ at $m_{D}=200 \mathrm{GeV}$ [10]. In this case, our numerical results are still far less than $6.4 \times 10^{-15} \mathrm{~cm}^{-2} \mathrm{~s}^{-1}$.

For the SSDM-2HBDM, the large Yukawa scale factors $R_{l}=10$ for charged leptons can significantly enhance the branching ratio of the $\tau^{+} \tau^{-}$annihilation channel when two DM particles can 

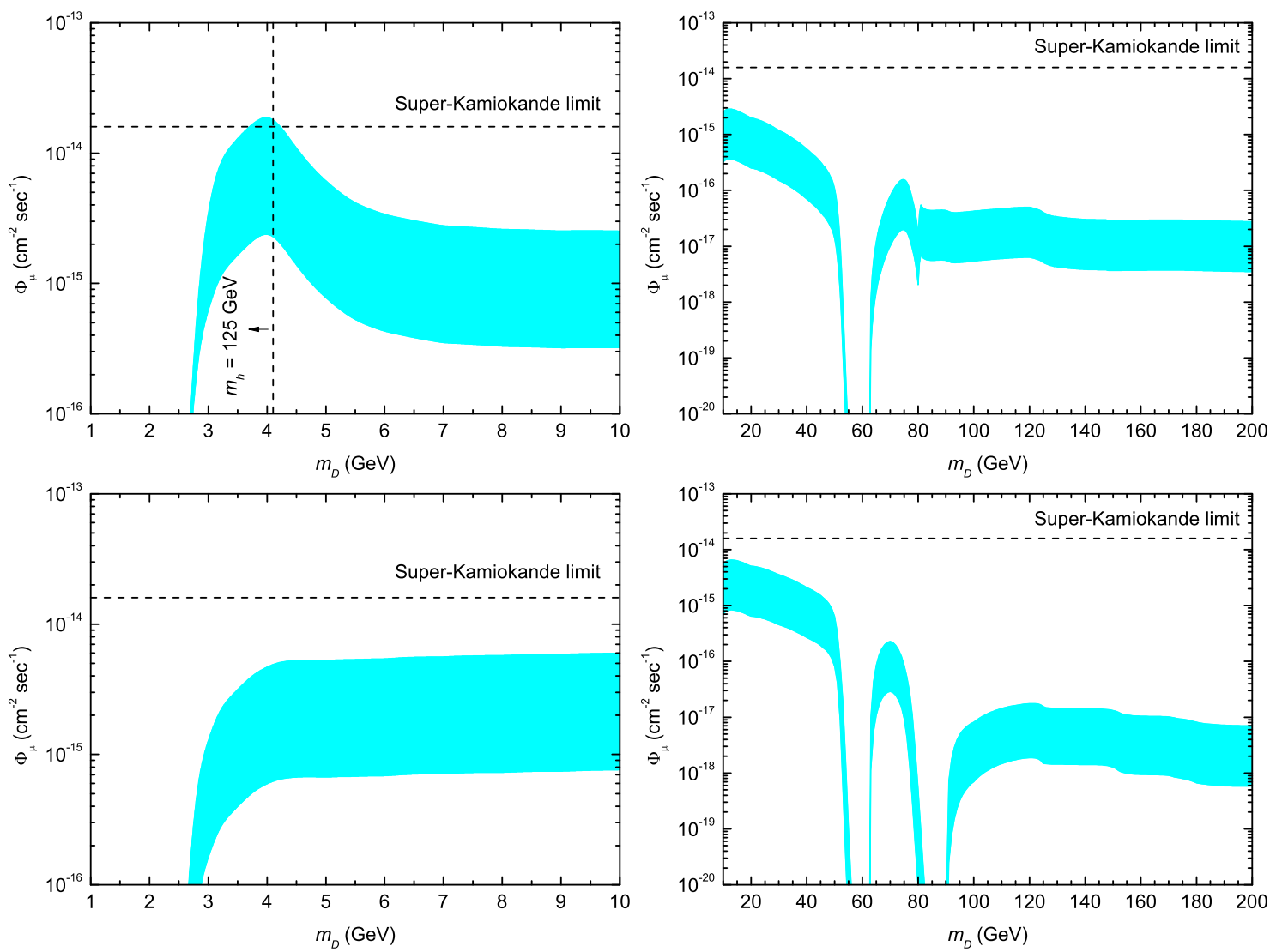

FIG. 4: The predicted neutrino induced upgoing muon fluxes for $1 \mathrm{GeV} \leq m_{D} \leq 200 \mathrm{GeV}$ in the SSDMSM (top row) and SSDM-2HBDM (bottom row). The dashed line denotes the Super-Kamiokande muon flux limit.

not annihilate into $W^{+} W^{-}\left(m_{D}<m_{W}\right)$. Since the produced muon event numbers from a pair of $\tau^{+} \tau^{-}$are far larger than those from $b \bar{b}$ and $c \bar{c}$. Therefore the SSDM-2HBDM with the enhanced $\tau^{+} \tau^{-}$branching ratio $\left(B_{\tau^{+} \tau^{-}} \simeq 53 \%\right.$ at $\left.m_{D}=10 \mathrm{GeV}\right)$ can give larger neutrino induced upgoing muon fluxes than those in the SSDM-SM even if the SSDM-2HBDM has the smaller $\sigma_{n}^{\text {SI }}$ as shown in Figs. 2 and 4. If $R_{l} \gg 10$, one will obtain a smaller $\lambda_{1, D}$ from the desired DM relic density which leads to a smaller $\sigma_{n}^{\text {SI }}$. In this case, the SSDM-2HBDM will produce smaller muon fluxes since $R_{l} \gg 10$ does not significantly enlarge $B_{\tau^{+} \tau^{-}}$. For $10 \mathrm{GeV} \leq m_{D} \leq 200 \mathrm{GeV}$, the predicted muon fluxes in the SSDM-2HBDM are far less than the Super-Kamiokande limit as shown in Fig. 4 (bottom right panel). Since $\sigma_{n}^{\text {SI }}$ in the SSDM-2HBDM may approach the current experimental upper bound through adjusting $R_{q}$, we can roughly evaluate the maximal muon fluxes from Figs. 2 and 4 (bottom right panels). We find that the maximal neutrino induced upgoing muon fluxes in 
the SSDM-2HBDM are still less than the Super-Kamiokande limit when $m_{D}>m_{W}$.

\section{Neutrino induced upgoing muon event rates in the IceCube}

The neutrino induced upgoing muons can also be detected by the neutrino telescope IceCube [11]. In this subsection, we use the following formula to calculate the neutrino induced upgoing muon event rates in the IceCube:

$$
\begin{aligned}
N_{\mu}= & \int_{E_{\mathrm{thr}}}^{m_{D}} d E_{\mu} A_{\mathrm{eff}}\left(E_{\mu}\right) \frac{\left\langle R\left(\cos \theta_{z}\right)\right\rangle}{2} \frac{1}{\rho\left(\alpha+\beta E_{\mu}\right)} \int_{E_{\mu}}^{m_{D}} d E_{v_{\mu}} \frac{d \Phi_{\nu_{\mu}}}{d E_{v_{\mu}}} \int_{E_{\mu}}^{E_{\nu_{\mu}}} d E_{\mu}^{\prime} \sum_{a=p, n} \frac{d \sigma_{v_{\mu}}^{a}\left(E_{v_{\mu}}, E_{\mu}^{\prime}\right)}{d E_{\mu}^{\prime}} \rho_{a} \\
& +\left(v_{\mu} \rightarrow \bar{v}_{\mu}\right),
\end{aligned}
$$

where $A_{\text {eff }}\left(E_{\mu}\right)$ and $E_{\text {thr }}^{\mathrm{IC}}=50 \mathrm{GeV}$ are the effective area and the threshold energy of the IceCube detector. To a good approximation, $A_{\text {eff }}\left(E_{\mu}\right)$ has a very simple functional form [47]

$$
\begin{aligned}
A_{\mathrm{eff}}\left(E_{\mu} \leq 10^{1.6} \mathrm{GeV}\right) & =0, \\
A_{\mathrm{eff}}\left(10^{1.6} \mathrm{GeV}<E_{\mu}<10^{2.8} \mathrm{GeV}\right) & =0.748\left[\log \left(E_{\mu} / \mathrm{GeV}\right)-1.6\right] \mathrm{km}^{2}, \\
A_{\mathrm{eff}}\left(E_{\mu} \geq 10^{2.8} \mathrm{GeV}\right) & =0.9+0.54\left[\log \left(E_{\mu} / \mathrm{GeV}\right)-2.8\right] \mathrm{km}^{2} .
\end{aligned}
$$

$R\left(\cos \theta_{z}\right)$ is a phenomenological angular dependence of the effective area for upgoing muons

$$
R\left(\cos \theta_{z}\right)=0.92-0.45 \cos \theta_{z},
$$

where $\theta_{z}$ is the zenith angle. Considering the change of the Sun direction, we average $R\left(\cos \theta_{z}\right)$ from $\cos \left(90^{\circ}\right)$ to $\cos \left(113.43^{\circ}\right)$ and derive $\left\langle R\left(\cos \theta_{z}\right)\right\rangle=1.01$. The factor of $1 / 2$ in Eq. (27) accounts for about $50 \%$ of the time that the Sun is below the horizon. For the ice, we take $\alpha=2.7 \times$ $10^{-3} \mathrm{~g}^{-1} \mathrm{GeV} \mathrm{cm}{ }^{2}, \beta=3.3 \times 10^{-6} \mathrm{~g}^{-1} \mathrm{~cm}^{2}, \rho_{p} \approx 5 / 9 N_{A} \rho$ and $\rho_{n} \approx 4 / 9 N_{A} \rho$ [45].

We use the above formulas to calculate the muon neutrino and muon anti-neutrino induced upgoing muon event rates as well as the background from atmosphere neutrinos in the IceCube. The atmosphere neutrino fluxes $d \Phi_{v_{\mu}} / d E_{v_{\mu}}\left(\cos \theta_{z}\right)$ can be found in Ref. [48]. For the atmosphere background, $\left\langle R\left(\cos \theta_{z}\right)\right\rangle d \Phi_{v_{\mu}} / d E_{v_{\mu}}$ in Eq. (27) should be replaced by $\left\langle R\left(\cos \theta_{z}\right) d \Phi_{v_{\mu}} / d E_{v_{\mu}}\left(\cos \theta_{z}\right)\right\rangle$. In order to reduce the background from atmosphere neutrinos, we require $E_{\text {thr }}^{\mathrm{IC}} \leq E_{\mu} \leq 200 \mathrm{GeV}$ and only consider the fluxes observed along the line of sight to the Sun within the $2^{\circ}$ half-angle cone [5]. Our numerical results have been shown in Fig. 5, It is found that the predicted muon event rates in the SSDM-SM and SSDM-2HBDM are less than the atmosphere background 10.2 $\mathrm{yr}^{-1}$. 

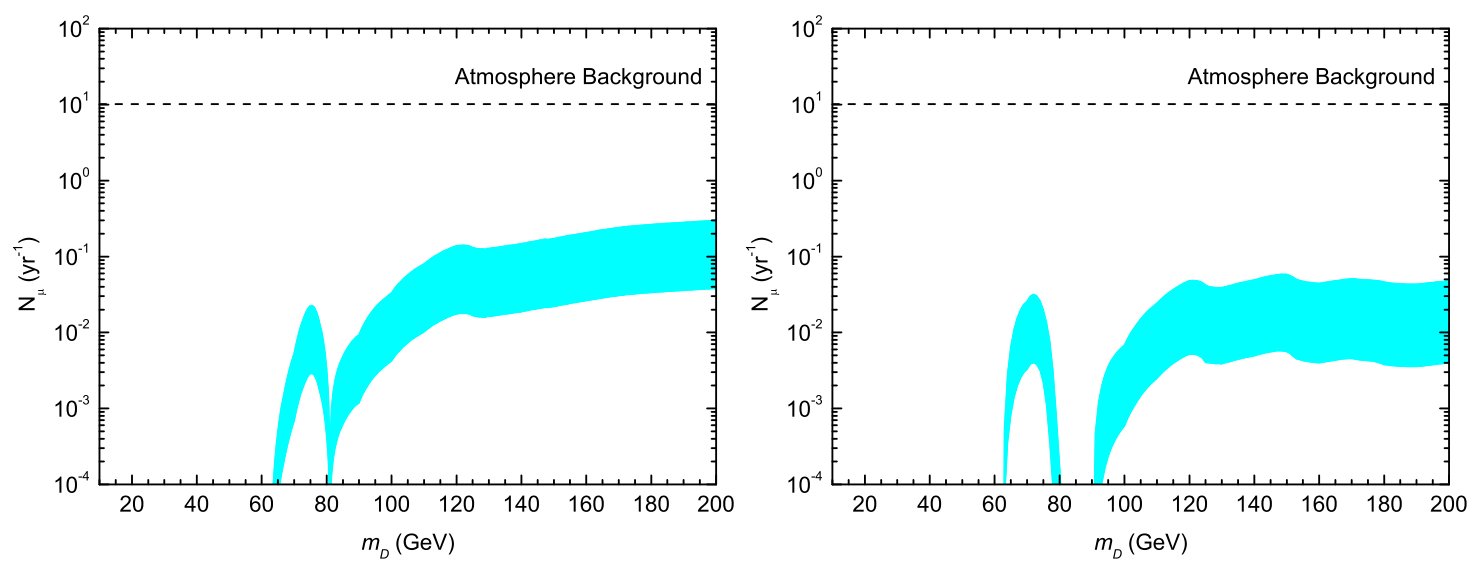

FIG. 5: The predicted muon event rates for $10 \mathrm{GeV} \leq m_{D} \leq 200 \mathrm{GeV}$ in the SSDM-SM (left panel) and SSDM-2HBDM (right panel). The dashed line denotes the atmosphere background in the IceCube.

\section{DISCUSSIONS AND CONCLUSIONS}

In terms of the observed DM abundance, we can derive the DM-Higgs couplings $\lambda$ in the SSDM-SM and $\lambda_{1, D}$ in the SSDM-2HBDM. If $\lambda^{2}$ and $\lambda_{1, D}^{2}$ are enlarged by $X$ times, the spinindependent DM-nucleon elastic scattering cross section $\sigma_{n}^{\text {SI }}$ in the SSDM-SM and SSDM2HBDM will be enlarged by the same times as shown in Eqs. (2) and (8). Since the DM relic density will be approximately suppressed by $X$ times, one thus needs to introduce new DM candidates. In terms of Eq. (13), one may find that the produced neutrino signals from the DM candidates $S$ and $S_{D}$ do not significantly change as the couplings $\lambda$ and $\lambda_{1, D}$ increase.

In conclusion, we have investigated the singlet scalar dark matter from direct detections and high energy neutrino signals via the solar DM annihilation in the SSDM-SM and SSDM-2HBDM. Firstly, we consider the uncertainties in the hadronic matrix elements and recalculate the spinindependent DM-nucleon elastic scattering cross section $\sigma_{n}^{\mathrm{SI}}$. It is found that the current DM direct detection experiments can exclude the $f \gtrsim 0.63$ region for $1 \mathrm{GeV} \leq m_{D} \leq 10 \mathrm{GeV}$ in the SSDM-SM. The latest XENON100 may exclude $7 \mathrm{GeV} \lesssim m_{D} \lesssim 52 \mathrm{GeV}$ and a narrow region $65 \mathrm{GeV} \lesssim m_{D} \lesssim 80 \mathrm{GeV}$ for $m_{h}=125 \mathrm{GeV}$ even if we take $f=0.26$. For the SSDM-2HBDM, we can adjust the Yukawa couplings to avoid the direct detection limits. Then we numerically calculate the neutrino fluxes from the DM annihilation in the Sun and the neutrino induced upgoing muon fluxes in the Super-Kamiokande and IceCube. The predicted muon fluxes in the region $3.7 \mathrm{GeV} \leq m_{D} \leq 4.2 \mathrm{GeV}$ and $f \gtrsim 0.65$ slightly exceed the Super-Kamiokande limit in the 
SSDM-SM. However, this exceeded region can be excluded by the CDMS (shallow-site data). We find that the SSDM-2HBDM can give larger muon fluxes than those in the SSDM-SM even if the SSDM-2HBDM has smaller $\sigma_{n}^{\mathrm{SI}}$. This is because that the large Yukawa scale factors $R_{l}=10$ for charged leptons can significantly enhance the branching ratio of the $\tau^{+} \tau^{-}$annihilation channel and the produced muon event numbers from a pair of $\tau^{+} \tau^{-}$are far larger than those from $b \bar{b}$ and $c \bar{c}$. For the allowed parameter space of the SSDM-SM and SSDM-2HBDM, the produced muon fluxes in the Super-Kamiokande and muon event rates in the IceCube are less than the experiment upper bound and atmosphere background, respectively. The large muon fluxes in $3 \mathrm{GeV} \lesssim m_{D} \lesssim 10$ $\mathrm{GeV}$ indicate that the future neutrino experiments can provide constraints on the SSDM-SM and SSDM-2HBDM.

\section{Acknowledgments}

This work is supported in part by the National Basic Research Program of China (973 Program) under Grants No. 2010CB833000; the National Nature Science Foundation of China (NSFC) under Grants No. 10821504 and No. 10905084; and the Project of Knowledge Innovation Program (PKIP) of the Chinese Academy of Science.

[1] G. Jungman, M. Kamionkowski and K. Griest, Phys. Rept. 267, 195 (1996).

[2] G. Bertone, D. Hooper and J. Silk, Phys. Rept. 405, 279 (2005).

[3] E. Komatsu et al. [WMAP Collaboration], Astrophys. J. Suppl. 192, 18 (2011) [arXiv:1001.4538 [astro-ph.CO]].

[4] V. Barger, W. Y. Keung, G. Shaughnessy and A. Tregre, Phys. Rev. D 76, 095008 (2007) [arXiv:0708.1325 [hep-ph]].

[5] J. Liu, P. f. Yin and S. h. Zhu, Phys. Rev. D 77, 115014 (2008) [arXiv:0803.2164[hep-ph]].

[6] D. Hooper, F. Petriello, K. M. Zurek and M. Kamionkowski, Phys. Rev. D 79, 015010 (2009) [arXiv:0808.2464 [hep-ph]].

[7] A. E. Erkoca, M. H. Reno and I. Sarcevic, Phys. Rev. D 80, 043514 (2009) [arXiv:0906.4364 [hep$\mathrm{ph}]]$.

[8] J. Ellis, K. A. Olive, C. Savage and V. C. Spanos, Phys. Rev. D 81, 085004 (2010) arXiv:0912.3137 
[hep-ph]].

[9] G. Wikstrom and J. Edsjo, JCAP 0904, 009 (2009) [arXiv:0903.2986 [astro-ph.CO]]; V. Niro, A. Bottino, N. Fornengo and S. Scopel, Phys. Rev. D 80, 095019 (2009) [arXiv:0909.2348 [hep-ph]]; J. Shu, P. f. Yin and S. h. Zhu, Phys. Rev. D 81, 123519 (2010) [arXiv:1001.1076 [hep-ph]]; A. L. Fitzpatrick, D. Hooper and K. M. Zurek, Phys. Rev. D 81, 115005 (2010) [arXiv:1003.0014[hep-ph]]; P. Agrawal, Z. Chacko, C. Kilic and R. K. Mishra, arXiv:1003.5905 [hep-ph]; V. Barger, J. Kumar, D. Marfatia and E. M. Sessolo, Phys. Rev. D 81, 115010 (2010) [arXiv:1004.4573][hep-ph]]; M. A. Ajaib, I. Gogoladze and Q. Shafi, Phys. Rev. D 83, 075017 (2011) [arXiv:1101.0835 [hep-ph]]; N. F. Bell and K. Petraki, JCAP 1104, 003 (2011) [arXiv:1102.2958 [hep-ph]].

[10] S. Desai et al. [Super-Kamiokande Collaboration], Phys. Rev. D 70, 083523 (2004) [Erratum-ibid. D 70, 109901 (2004)] [arXiv:hep-ex/0404025].

[11] J. Ahrens et al., IceCube Preliminary Design Document (2001); J. Ahrens et al. [IceCube Collaboration], Astropart. Phys. 20, 507 (2004) [arXiv:astro-ph/0305196].

[12] V. Silveira and A. Zee, Phys. Lett. B 161, 136 (1985); J. McDonald, Phys. Rev. D 50, 3637 (1994) arXiv:hep-ph/0702143]; X. G. He, T. Li, X. Q. Li, J. Tandean and H. C. Tsai, Phys. Rev. D 79, 023521 (2009) [arXiv:0811.0658 [hep-ph]]; Phys. Lett. B 688, 332 (2010) [arXiv:0912.4722 [hepph]]; S. Profumo, L. Ubaldi and C. Wainwright, Phys. Rev. D 82, 123514 (2010) [arXiv:1009.5377 [hep-ph]].

[13] C. P. Burgess, M. Pospelov and T. ter Veldhuis, Nucl. Phys. B 619, 709 (2001) [arXiv:hep-ph/0011335].

[14] M. Gonderinger, Y. Li, H. Patel and M. J. Ramsey-Musolf, JHEP 1001, 053 (2010) arXiv:0910.3167 [hep-ph]].

[15] S. Andreas, T. Hambye and M. H. G. Tytgat, JCAP 0810, 034 (2008) arXiv:0808.0255 [hepph]]; A. Bandyopadhyay, S. Chakraborty, A. Ghosal and D. Majumdar, JHEP 1011, 065 (2010) [arXiv:1003.0809 [hep-ph]]; S. Andreas, C. Arina, T. Hambye, F. -S. Ling and M. H. G. Tytgat, Phys. Rev. D 82, 043522 (2010) [arXiv:1003.2595[hep-ph]]; V. Barger, M. McCaskey and G. Shaughnessy, Phys. Rev. D 82, 035019 (2010) [arXiv:1005.3328 [hep-ph]]; C. Arina and M. H. G. Tytgat, JCAP 1101, 011 (2011) [arXiv:1007.2765 [astro-ph.CO]].

[16] W. L. Guo and Y. L. Wu, JHEP 1010, 083 (2010) [arXiv:1006.2518 [hep-ph]], and references therein.

[17] Y. L. Wu and Y. F. Zhou, Sci. China G51, 1808 (2008) [arXiv:0709.0042 [hep-ph]]; Int. J. Mod. Phys. A 23, 3304 (2008) [arXiv:0711.3891 [hep-ph]]. 
[18] W. L. Guo, Y. L. Wu and Y. F. Zhou, Phys. Rev. D 81, 075014 (2010) [arXiv:1001.0307[hep-ph]].

[19] W. L. Guo, Y. L. Wu and Y. F. Zhou, Phys. Rev. D 82, 095004 (2010) [arXiv:1008.4479 [hep-ph]]; W. L. Guo, L. M. Wang, Y. L. Wu, Y. F. Zhou and C. Zhuang, Phys. Rev. D 79, 055015 (2009) [arXiv:0811.2556[hep-ph]].

[20] ATLAS Collaboration, ATLAS-CONF-2012-093; CMS Collaboration, CMS-PAS-HIG-12-020.

[21] We miss a factor $1 / 2$ for $a_{q}$ in Ref. [16]. Therefore the predicted direct detection cross sections in Ref. [16] correspond to the $f=0.7$ case in this paper.

[22] J. Giedt, A. W. Thomas and R. D. Young, Phys. Rev. Lett. 103, 201802 (2009) arXiv:0907.4177 [hep-ph]].

[23] R. Bernabei et al. [DAMA Collaboration], Eur. Phys. J. C 56, 333 (2008) [arXiv:0804.2741 [astro$\mathrm{ph}]]$.

[24] C. E. Aalseth et al. [CoGeNT Collaboration], Phys. Rev. Lett. 106, 131301 (2011) [arXiv:1002.4703 [astro-ph.CO]].

[25] D. Hooper, J. I. Collar, J. Hall and D. McKinsey, Phys. Rev. D 82, 123509 (2010) [arXiv:1007.1005 [hep-ph]].

[26] Z. Ahmed et al. [CDMS-II Collaboration], Phys. Rev. Lett. 106, 131302 (2011) arXiv:1011.2482 [astro-ph.CO]]; Science 327, 1619 (2010) [arXiv:0912.3592 [astro-ph.CO]].

[27] D. S. Akerib et al. [CDMS Collaboration], Phys. Rev. D 82, 122004 (2010) [arXiv:1010.4290] [astroph.CO]].

[28] G. Angloher et al., Astropart. Phys. 18, 43 (2002).

[29] S. T. Lin et al. [TEXONO Collaboration], Phys. Rev. D 79, 061101 (2009) [arXiv:0712.1645 [hepex]].

[30] E. Aprile et al. [XENON100 Collaboration], arXiv:1207.5988 [astro-ph.CO].

[31] J. Cooley, SLAC seminar on Dec. 17, 2009; L. Hsu, Fermilab seminar on Dec. 17, 2009.

[32] Elena Aprile, XENON1T: a ton scale Dark Matter Experiment, presented at UCLA Dark Matter 2010, February 26, 2010. The XENON1000 project in China has been supported in part by the National Basic Research Program of China (973 Program).

[33] M. E. Pospelov, Phys. Rev. D 56, 259 (1997) [arXiv:hep-ph/9611422]; Y. Zhang, H. An, X. Ji and R. N. Mohapatra, Phys. Rev. D 76, 091301 (2007) [arXiv:0704.1662 [hep-ph]]; A. Maiezza, M. Nemevsek, F. Nesti and G. Senjanovic, Phys. Rev. D 82, 055022 (2010) [arXiv:1005.5160 [hep-ph]].

[34] J. Edsjo, WimpSim Neutrino Monte Carlo, http://www.physto.se/ edsjo/wimpsim/; M. Blennow, 
J. Edsjo and T. Ohlsson, JCAP 0801, 021 (2008) [arXiv:0709.3898 [hep-ph]].

[35] T. Sjostrand, S. Mrenna and P. Z. Skands, JHEP 0605, 026 (2006) [arXiv:hep-ph/0603175].

[36] J. Edsjo, Nusigma 1.16.

[37] P. Gondolo, J. Edsjo, P. Ullio, L. Bergstrom, M. Schelke and E. A. Baltz, JCAP 0407, 008 (2004) [arXiv:astro-ph/0406204].

[38] J. N. Bahcall, A. M. Serenelli and S. Basu, Astrophys. J. 621, L85 (2005) [arXiv:astro-ph/0412440].

[39] F. P. An et al. [DAYA-BAY Collaboration], Phys. Rev. Lett. 108, 171803 (2012) [arXiv:1203.1669 [hep-ex]].

[40] D. V. Forero, M. Tortola and J. W. F. Valle, arXiv:1205.4018 [hep-ph].

[41] K. Griest and D. Seckel, Nucl. Phys. B 283, 681 (1987) [Erratum-ibid. B 296, 1034 (1988)].

[42] A. Gould, Astrophys. J. 321, 560 (1987).

[43] D. Feldman, Z. Liu and P. Nath, Phys. Rev. D 79, 063509 (2009) [arXiv:0810.5762 [hep-ph]]; M. Ibe, H. Murayama and T. T. Yanagida, Phys. Rev. D 79, 095009 (2009) [arXiv:0812.0072 [hep-ph]]; W. L. Guo and Y. L. Wu, Phys. Rev. D 79, 055012 (2009) [arXiv:0901.1450 [hep-ph]].

[44] T. K. Gaisser and T. Stanev, Phys. Rev. D 30, 985 (1984).

[45] L. Covi, M. Grefe, A. Ibarra and D. Tran, JCAP 1004, 017 (2010) [arXiv:0912.3521][hep-ph]].

[46] M. Mori et al. [KAMIOKANDE Collaboration], Phys. Rev. D 48, 5505 (1993).

[47] M. C. Gonzalez-Garcia, F. Halzen and S. Mohapatra, Astropart. Phys. 31, 437 (2009) [arXiv:0902.1176 [astro-ph.HE]].

[48] M. Honda, T. Kajita, K. Kasahara, S. Midorikawa and T. Sanuki, Phys. Rev. D 75, 043006 (2007) [arXiv:astro-ph/0611418]. 